\title{
Preface to high performance computing applied to computational problems in science and engineering
}

\author{
Raquel Cortina · F.J. Martínez-Zaldívar • \\ Antonio M. Vidal · Jesús Vigo-Aguiar
}

Published online: 16 August 2012

(C) Springer Science+Business Media, LLC 2012

As a part of the 11th International Conference on "Computational and Mathematical Methods in Science and Engineering" (CMMSE 2011), the Minisymposium "High Performance Computing (HPC) applied to Computational Problems in Science and Engineering" took place in Benidorm on 26 and 27 of June. It brought together over 100 researchers associated within the field of HPC, who participated in the various oral and poster sessions.

In last years, the number of scientific contributions and research projects related to the HPC based on the use of parallel computers (multicore processors, Graphics Processing Units-GPU_or clusters of computers) has significantly increased. This phenomenon has occurred in almost all engineering fields that require intensive computing.

Heterogeneous parallel computers based on manycore (GPU) and multicore microprocessors are fascinating tools and represent a quantitative leap in the development of high performance hardware. Probably it would be impossible to go back,

R. Cortina ( $\varangle)$

Universidad de Oviedo, Oviedo, Asturias, Spain

e-mail: raquel@uniovi.es

F.J. Martínez-Zaldívar

iTEAM, Universitat Politècnica de València, Valencia, Spain

e-mail: fjmartin@dcom.upv.es

A.M. Vidal

INCO2, Universitat Politècnica de València, Valencia, Spain

e-mail: avidal@dsic.upv.es

J. Vigo-Aguiar

Universidad de Salamanca, Salamanca, Spain

e-mail: jvigo@usal.es 
and the near future cannot be imagined without GPU or multicore technology. However, to give accurate opinions and ensure their usefulness, it is essential to carry out a quantitative performance analysis. It should show the ranges and working conditions for which the attained benefits are really important, together with a quantitative assessment of those benefits. A realistic approach to the performance of multicore/manycore in a particular field involves identifying the problems that can be solved with these tools, defining which benchmarks will be used in performance analysis and setting evaluation metrics.

This special issue offers to the readers of Journal of Supercomputing, a selection of papers presented in the Minisymposium. All papers in this special issue have passed through a new revision process. The volume collects 16 of the most significant contributions presented in the Minisymposium and aims to provide an overview of current research in the field of HPC and its applications. The papers show efficient implementations or performance evaluations in sequential and parallel systems as multicore/manycore and distributed memory architectures in fields as:

- Digital Signal Processing: two papers, computing 2D Discrete Wavelet transform in shared memory systems and GPUs, and computing the Fast Multipole Method for solving acoustics scattering problems on distributed-memory GPGPUs.

- Electromagnetics: implementation of Finite Difference Time-Domain method for transient analysis and the Finite Element Method for Electrostatics in CPU and GPU.

- Algorithmic and Numerical Linear Algebra problems: with three papers showing threaded implementations of the quadratic biblending algorithm, implementations of the BiConjugate Gradient Method on GPUs solving complex and/or nonsymmetric linear systems, and extending a library of hierarchical tiling arrays to support sparse data partitioning using MPI.

- Biotechnology: solving the problem of Multiple Sequence Alignment on new parallel systems using multithreading and MPI programming.

- Video processing: with two papers, the first one showing a GPU implementation of the H.264/AVC Motion Estimation and the second one with a scalable video segmentation application detecting the shot boundary in shared memory and distributed memory systems.

- Performance evaluation of parallel systems and computational kernels: with three papers showing the performance of sparse matrix products using Unified Parallel C (UPC), studying and redefining the isoefficiency function in parallel systems executing balanced or unbalanced workloads, and finally using the FFT as benchmark to study the influence of memory access patterns in the performance of a GPU.

- Cryptographic protocols: with a paper showing the performance of a scalable server for key distribution using an efficient secure multicast protocol in multicore and GPU systems.

- Distributed file systems: managing metadata in distributed file systems with twolevel hash/table approach.

- Simulation: using the read-copy update (RCU) synchronization method to improve distributed crowd simulations.

- Automatic code generation: using LaTeX specifications to generate efficient parallel code for homogeneous or heterogeneous architectures. 
We want to express our gratitude to all participants for their contributions and for making the Minisymposium an interesting and pleasant event. We sincerely wish to thank the authors and the reviewers who helped us in doing the editorial work. Their efforts, corrections, and suggestions for improvement made it possible to publish scientific papers of high quality.

We would also like to acknowledge the Spanish Office of Science, for its support to the Spanish network "CAPAP-H (HPC in Heterogeneous Architectures, TIN201012011-E)". Last, we also greatly appreciate the help and encouragement received from Professor J. Ranilla in editing this special issue. 